\title{
Acute unilateral hydrocephalus resulting from oedematous occlusion of foramen of Monro: complication of intraventricular surgery
}

\author{
THOMAS H. MILHORAT, MARY K. HAMMOCK, \\ AND DAVID L. BRECKBILL \\ From the Departments of Neurosurgery and Radiology, \\ Children's Hospital National Medical Center, Washington, D.C., 20009, \\ and the Branch of Surgical Neurology, NINDS, Bethesda, Maryland, 20014, U.S.A.
}

SYNOPSIS Transcerebral removal of a small choroid plexus papilloma in the region of the foramen of Monro was followed within 48 hours by severe and progressive unilateral hydrocephalus. Acute unilateral hydrocephalus is a hitherto unrecognized complication of intraventricular surgery.

Sudden obstruction of the cerebrospinal fluid pathways may be followed within hours by a significant dilatation of the system proximal to the block. This phenomenon, which can be readily demonstrated in animals by obstructing the fourth ventricle with an inflatable balloon (Milhorat et al., 1970), results in a rapid evolution of pathological changes including disruption of the ventricular ependyma and oedema of the subependymal white matter (Clark and Milhorat, 1970). In humans, acute hydrocephalus may be associated with any of the following conditions: spontaneous subarachnoid haemorrhage, acute head injury, traumatic haemorrhage into the posterior fossa, acute exudative meningitis, intracerebral or intracerebellar haemorrhage, decompensation of tumours bordering the ventricular system, and obstruction of functioning ventricular shunts (Milhorat, 1970, 1971a, 1972).

Although lesions that obstruct one foramen of Monro are known to produce chronic enlargement of the ipsilateral lateral ventricle (Cushing, 1908; Dott, 1927; Cairns et al., 1947; Alexander and Botterell, 1949), so far as can be determined an example of acute unilateral hydrocephalus has yet to be described. The purpose of this communication is to report such a case and to call

(Accepted 10 March 1975.) attention to a life-threatening but easily treatable complication of intraventricular surgery.

\section{CASE REPORT}

A 10 year old male who had been previously well was admitted to Children's Hospital National Medical Center for evaluation of morning headaches of three weeks duration. The headaches were described as generalized and non-throbbing and were usually relieved by lying flat. With each attack, the patient experienced a sensation of 'lightheadedness', and on several occasions he complained of double-vision and an upset stomach. Physical examination revealed no findings other than mild bilateral papilloedema. Skull radiographs, brain scan, and carotid angiograms were unremarkable. Computerized axial tomography (EMI scan) and pneumoencephalography revealed selective dilatation of the frontal horn of the left lateral ventricle but no other obvious findings. The patient was started on acetazolamide (Diamox) and intermittent doses of dexamethasone and was followed closely by his pediatrician, an ophthalmologist, and a paediatric neurologist.

Three months later, because of recurring headaches, the patient was readmitted to the hospital. The physical examination was unchanged. A repeat pneumoencephalogram demonstrated persistent dilatation of the left frontal horn and evidence of a small filling defect in the region of the left foramen of Monro (Figure). Several days later, the patient underwent a left frontal craniotomy.

Using a standard transcerebral approach, the left 

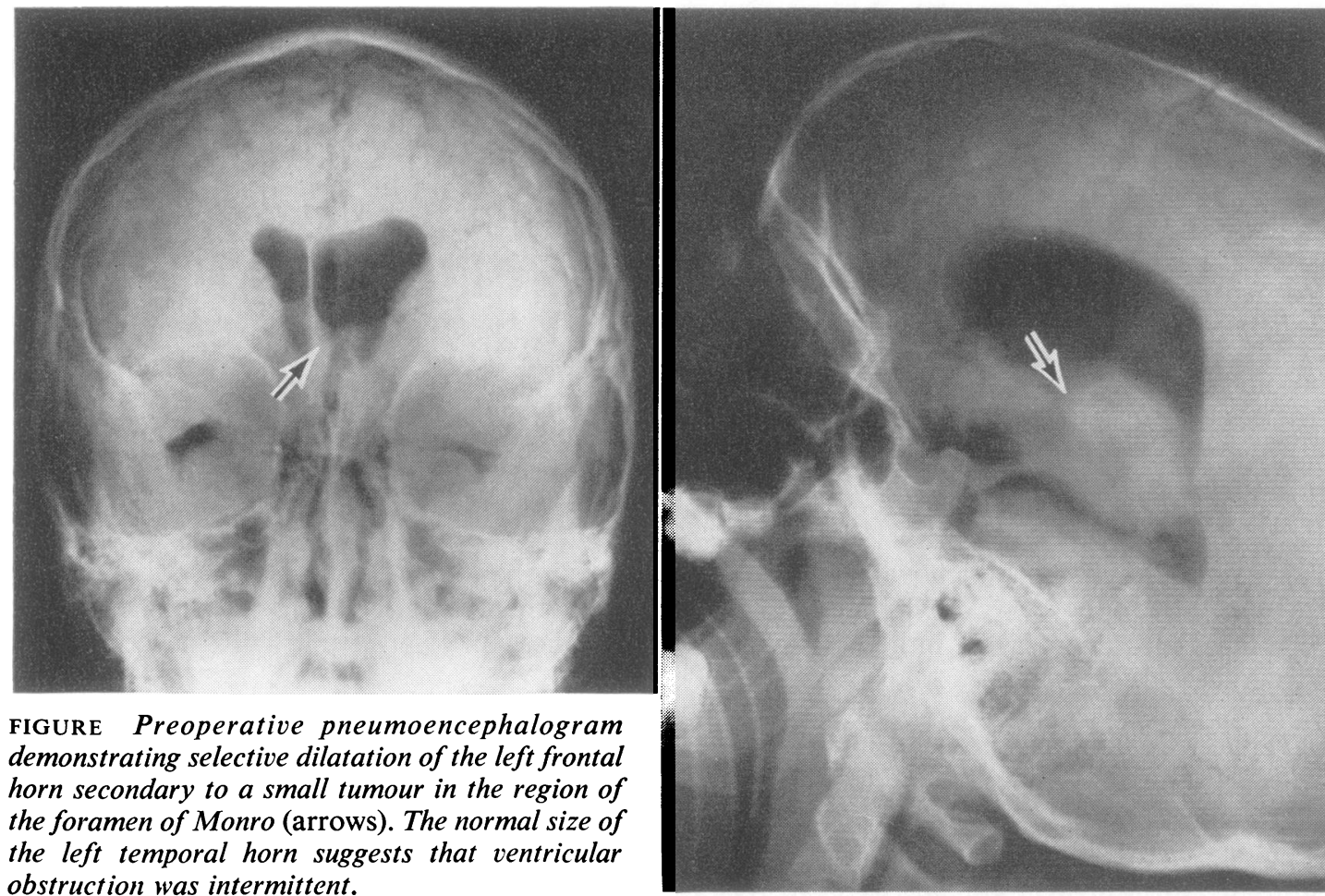

lateral ventricle was entered through a linear incision in the middle frontal gyrus. This exposed a peasized tumour situated within the orifice of the left foramen of Monro that was pedunculated, mobile, and attached to the choroid plexus by a vascular stalk. The stalk was secured with several silver clips and the tumour was excised. After reinflating the ventricles with sterile saline, the cerebral incision was closed with fine silk sutures (Milhorat, 1971b). Pathological examination of the submitted tumour specimen revealed it to be a choroid plexus papilloma.

After surgery, the patient awoke promptly. The next morning, he was alert and without symptoms except for mild bifrontal headache. On the morning of the second postoperative day, the patient was found to be somewhat less responsive. He refused to take meals or leave his bed and insisted on being 'left alone'. Throughout the day, his blood pressure rose gradually from $100 / 60$ to $160 / 100 \mathrm{mmHg}$ and his pulse fell from 80-110 to 56-60 per min. By midafternoon, he was noted to be generally somnolent but arousable. Intravenous fluids were administered, and dexamethasone in doses of $4 \mathrm{mg}$ every six hours was begun. Shortly after 8 p.m., the patient's con-

dition suddenly deteriorated. He became deep stuporous and could be aroused only by painfuil or stimuli. Examination revealed complete paralysis of the left oculomotor nerve, and weakness and decorticate posturing of the right upper extremity. A tentative diagnosis of intracranial haemorrhage or oedema was made and the patient was rushed to the operating room for removal of his bone plate.

During preparations for surgery, the patient suffered a respiratory arrest. Prompt resuscitative measures were instituted, and under less than optimum conditions the bone plate was removed. After establishing that the patient was not suffering from an extradural haematoma, the dural incision was reopened. Examination of the brain revealed marked tension of the left frontal lobe. The cerebral incision was found to be well-approximated and there was no evidence of cortical necrosis or oedema. On the basis of these findings, it was concluded that the patient was suffering from an intraventricular $D$ haematoma and a 16 gauge ventricular needle was passed into the left frontal horn. At a depth of two centimetres, the ventricle was entered. This resulted in the immediate release of a forceful stream of $\tilde{O}$ clear, colourless cerebrospinal fluid under high $\mathrm{c}$ 
pressure. Over a period of five to 10 minutes, approximately $100 \mathrm{ml}$ of fluid was drained, resulting in great relaxation of the brain. At this point, the patient began breathing spontaneously. The ventricular needle was replaced with a silastic catheter arranged to an external drainage bottle and the scalp was closed in a single layer with heavy silk.

Postoperatively, the patient recovered uneventfully. The drainage system was set at an elevation of $10 \mathrm{~cm}$ saline, and over the first four days, the ventricular output varied from $85-200 \mathrm{ml}$ per day. On the fifth and sixth postoperative days, attempts to clamp the ventricular catheter were followed within two to four hours by severe bifrontal headache. Ventricular pressures, measured at the time of unclamping, ranged from $25-35 \mathrm{~cm}$ saline. On the seventh postoperative day, a pneumoventriculogram was performed through the ventricular catheter. This revealed complete obstruction of the left foramen of Monro and no passage of air out of the left lateral ventricle in spite of numerous manipulations of the head. Over the next few days the patient tolerated intermittent clamping of the catheter increasingly well. On the twelfth postoperative day, a pneumoencephalogram revealed that the continuity of the ventricular system had been reestablished. The ventricular catheter was subsequently removed. At the time of discharge, one week later, the patient was fully ambulant and without symptoms. Examination revealed a dense right homonymous hemianopsia and a resolving left third nerve palsy.

Six months postoperatively, the patient was electively admitted to the hospital for insertion of an acrylic bone plate. Physical examination revealed complete resolution of the left third nerve palsy but persistence of a dense right homonymous hemianopsia. Visual acuity was $20 / 25$ bilaterally. These findings were essentially unchanged at follow-up one year after operation.

\section{DISCUSSION}

Although a clinical example of acute unilateral hydrocephalus has not been previously reported, the rapidity with which the human ventricular system can dilate after pathological obstruction of the third ventricle (Milhorat, 1971a), aqueduct of Sylvius (Lang, 1969), and fourth ventricle (Milhorat, 1970) is well documented. In the case reported here, acute unilateral hydrocephalus occurred as an unexpected complication 48 hours after the removal of a small choroid plexus papilloma in the region of the foramen of Monro. Since it was subsequently established that the foraminal occlusion was transient (resolving spontaneously within 12 days), the most likely cause was local tissue oedema. That the obstructing lesion was not an intraventricular clot was suggested by the finding of clear, non-xanthochromic fluid within the obstructed ventricle, and the failure of postoperative air studies to demonstrate a filling defect in the region of the foramen of Monro. The moderate enlargement of the affected ventricle at the time of surgery and the use of a cerebral suturing technique, which prevented the escape of cerebrospinal fluid from the obstructed ventricle, may have contributed to the rapid development of symptoms in this case.

The clinical manifestations of acute unilateral hydrocephalus were of particular interest. The initial symptoms were those of increasing intracranial tension: generalized headache, deepening stupor, rising blood pressure, and falling pulse. These were followed by focal signs indicative of transtentorial herniation: ipsilateral third nerve paralysis and contralateral hemiparesis. These signs and symptoms may be associated with any rapidly expanding hemispheral lesion and the correct diagnosis, acute unilateral hydrocephalus, was made only after emergency reoperation failed to disclose evidence of extradural or intraventricular bleeding. In retrospect, it is possible to conclude that rapid dilatation of the obstructed lateral ventricle resulted in acute herniation of the ipsilateral temporal lobe through the tentorial notch. Permanent contralateral homonymous hemianopsia after recovery can be similarly explained as a consequence of entrapment of the ipsilateral posterior cerebral artery.

Acute unilateral hydrocephalus should be considered as a possible complication in any patient exhibiting focal signs of a rapidly expanding intracranial mass following intraventricular surgery. Whereas extradural bleeding, intraventricular haemorrhage, and cerebral oedema are the most likely considerations in such circumstances, it is a simple matter to perform a ventricular tap or otherwise explore the cerebral incision at the time of reoperation. In this case, simple external drainage resulted in a prompt resolution of symptoms and an eventual cure.

Finally, it should be kept in mind that chronic enlargement of one lateral ventricle may occur after certain intraventricular operations as 
a consequence of ependymitis and delayed scarring of the foramen of Monro. Alexander and Botterell (1949) were the first to call attention to this complication and described a clinical syndrome characterized by intermittent headache, confusion, and contralateral hemiparesis. With the increasing availability of computerized axial tomography, it is anticipated that this noninvasive procedure will be useful in screening any patient who fails to follow the expected course of recovery after intraventricular surgery. The diagnosis of chronic unilateral hydrocephalus is important, since a ventricular shunting procedure or an operation to create an artificial opening through the septum pellucidum is usually curative (Dott, 1927; Alexander and Botterell, 1949; Milhorat, 1972).

\section{REFERENCES}

Alexander, E., Jr, and Botterell, E. H. (1949). Unilateral hydrocephalus resulting from occlusion of foramen of Monro. Complication of radical removal of brain abscess. Journal of Neurosurgery, 6, 197-206.
Cairns, H., Daniel, P., Johnson, R. T., and Northcroft, G. E. (1947). Localized hydrocephalus following penetrating wounds of the ventricle. British Journal of Surgery, War Surgery Suppl. 1, 187-197.

Clark, R. G., and Milhorat, T. H. (1970). Experimental hydrocephalus. Part 3. Light microscopic findings in acute and subacute obstructive hydrocephalus in the monkey. Journal of Neurosurgery, 32, 400-413.

Cushing, H. (1908). Surgery of the head. In Surgery, Its Principles and Practice, pp. 142-143. Edited by W. W. Keen. Saunders: Philadelphia.

Dott, N. M. (1927). A case of left unilateral hydrocephalus in an infant. Operation-cure. Brain, 50, 548-560.

Lang, E. K. (1969). Acute hydrocephalus secondary to occlusion of the aqueduct by a bullet. Journal of the Louisiana Medical Society, 121, 167-169.

Milhorat, T. H. (1970). Acute hydrocephalus. New England Journal of Medicine, 283, 857-859.

Milhorat, T. H. (1971a). Intracerebral hemorrhage, acute hydrocephalus and systemic hypertension. Journal of the American Medical Association, 218, 221-225.

Milhorat, T. H. (1971b). Closure of cerebral incisions following intraventricular operations. Journal of Neurosurgery, 35, 108-111.

Milhorat, T. H. (1972). Hydrocephalus and the Cerebrospinal Fluid. Williams and Wilkins: Baltimore.

Milhorat, T. H., Clark, R. G , and Hammock, M. K. (1970). Experimental hydrocephalus. Part 2. Gross pathological findings in acute and subacute obstructive hydrocephalus $\infty_{\infty}$ in the dog and monkey. Journal of Neurosurgery, 32, 390 399. 